\title{
Correction to: Modulation of miRNA in Oxidative Stress-Induced Cardiac Remodeling
}

Sudhiranjan Gupta

\section{Correction to:}

Chapter 3 in: S. Chakraborti et al. (eds.), Modulation of Oxidative Stress in Heart Disease, https://doi.org/10.1007/978-981-13-8946-7_3

The figure legends of Figs. 3.2 and 3.3 of this chapter were swapped inadvertently and published with errors. The correct presentation is given here. 
Fig. 3.2 Schematic presentation of ROSmediated miRNA modulation in cardiac remodeling. ROS can modulate the miRNAs through posttranscriptional regulation of NRF2 and Sirt2 mRNAs. Green arrow indicates upregulatory pattern and red arrow indicated downregulatory pattern

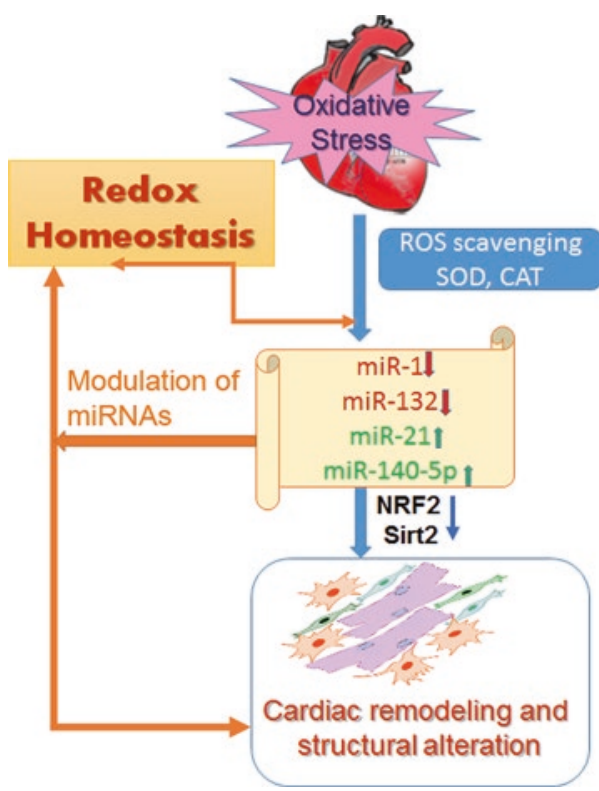



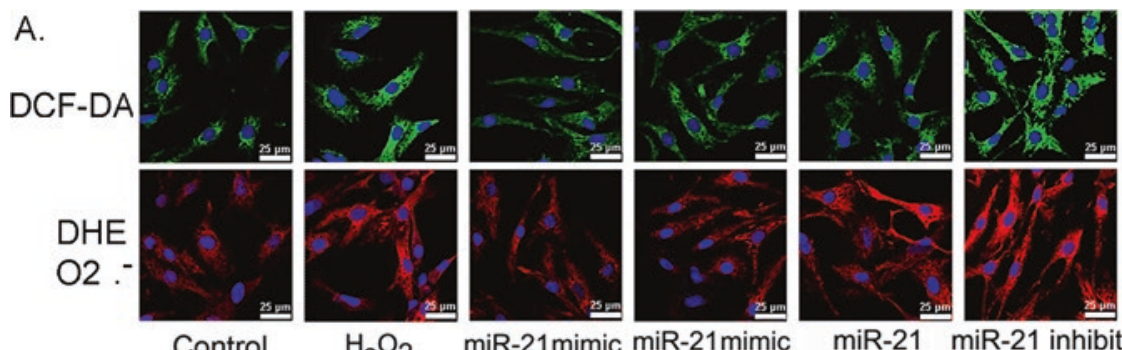

$\mathrm{H}_{2} \mathrm{O}_{2}$

miR-21mimic miR-21mimic

$+\mathrm{H}_{2} \mathrm{O}_{2}$

miR-21 miR-21 inhibitor

PXentrol

inhibitor $\quad+\mathrm{H}_{2} \mathrm{O}_{2}$

B.

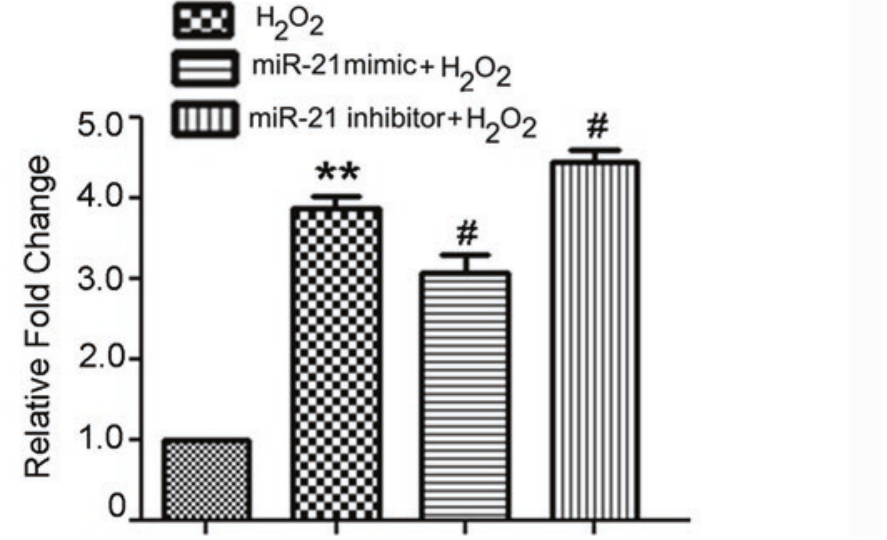

Fig. 3.3 Overexpression of miR-21 attenuates $\mathrm{H}_{2} \mathrm{O}_{2}$-induced $\mathrm{ROS}$ level in neonatal cardiomyocytes. The ROS level was measured in transfected neonatal cardiomyocytes with miR-21 mimetic and inhibitor followed by $\mathrm{H}_{2} \mathrm{O}_{2}$ treatment for $24 \mathrm{~h}$ by confocal microscopy and fluorimetry. (a) Representative confocal microscopy images of cardiomyocytes stained with DCFH-DA and DHE, respectively, showing the activity of $\mathrm{H}_{2} \mathrm{O}_{2}$ and $\mathrm{O}_{2}^{-}$. (b) Effect of miR-21 mimetic and inhibitor on generation of ROS in cardiomyocytes treated with $\mathrm{H}_{2} \mathrm{O}_{2}$ by fluorimetry. The data presented are mean \pm SE. $* * P<0.01$ vs. control, ${ }^{\#} P<0.05$ vs. $\mathrm{H}_{2} \mathrm{O}_{2}$ treatment $(n=3)$. (Adopted from Wei $\mathrm{C}$, Li L, Kim IK, Sun P, Gupta S. Free Radic Res. 2014, Reference 28) 